\title{
Quantum phase transitions in a quasi-one-dimensional Ising quantum magnet in transverse fields
}

\author{
Xiaowen Zhang, Zheng He, Yiqing Hao, Yao Shen, Shoudong Shen, \\ Yu Feng, Guangyong Xu, Nicholas P. Butch, Gøran J. Nilsen, \\ Wenbin Wang, and Jun Zhao
}

\section{Published version information}

Citation: X Zhang et al. Quantum phase transitions in a quasi-one-dimensional Ising quantum magnet in transverse fields. Phys Rev B 103, no. 14 (2021): 144405

DOI: $10.1103 /$ PhysRevB.103.144405

This version is made available in accordance with publisher policies. Please cite only the published version using the reference above. This is the citation assigned by the publisher at the time of issuing the APV. Please check the publisher's website for any updates. 


\title{
Quantum phase transitions in a quasi-one-dimensional Ising quantum magnet in transverse fields
}

\author{
Xiaowen Zhang, ${ }^{1}$ Zheng He, ${ }^{1}$ Yiqing Hao, ${ }^{1}$ Yao Shen, ${ }^{1}$ Shoudong Shen, ${ }^{1}$ Yu Feng $\odot,{ }^{1}$ Guangyong Xu, ${ }^{2}$ Nicholas P. Butch, ${ }^{2}$ \\ Gøran J. Nilsen, ${ }^{3}$ Wenbin Wang, ${ }^{4, *}$ and Jun Zhao ${ }^{1,5,6, \dagger}$ \\ ${ }^{1}$ State Key Laboratory of Surface Physics and Department of Physics, Fudan University, Shanghai 200433, China \\ ${ }^{2}$ NIST Center for Neutron Research, National Institute of Standards and Technology, Gaithersburg, Maryland 20899, USA \\ ${ }^{3}$ ISIS Facility, Rutherford Appleton Laboratory, STFC, Chilton, Didcot, Oxon OX11 OQX, United Kingdom \\ ${ }^{4}$ Institute of Nanoelectronic Devices and Quantum Computing, Fudan University, Shanghai 200433, China \\ ${ }^{5}$ Shanghai Qi Zhi Institute, Shanghai 200232, China \\ ${ }^{6}$ Collaborative Innovation Center of Advanced Microstructures, Nanjing 210093, China
}

(Received 4 December 2020; accepted 22 March 2021; published 2 April 2021; corrected 15 April 2021)

\begin{abstract}
We report neutron scattering and thermodynamic measurements of the quasi-one-dimensional Ising magnet $\mathrm{SrCo}_{2} \mathrm{~V}_{2} \mathrm{O}_{8}$ under transverse fields along the tetragonal $a / b$ direction. Our experiments reveal a Néel-type magnetic order in zero field, which successively changes into a coplanar antiferromagnetic order in applied fields. The detailed evolution of the noncollinear magnetic order in magnetic fields can be understood by considering the competition between the intrinsic magnetic interactions, uniform transverse field, and effective staggered field. Moreover, a series of gapped discrete confined-spinon modes are observed in zero field. With increasing field, the spin gap progressively softens, reaching a minimum value at $\sim 7 \mathrm{~T}$ where the Néel-type magnetic ordering moment is completely suppressed. This corresponds to the three-dimensional quantum critical point (QCP). The implications for multiple QCPs in this class of materials are discussed.
\end{abstract}

DOI: 10.1103/PhysRevB.103.144405

\section{INTRODUCTION}

A quantum criticality typically emerges when tuning a continuous phase transition to zero temperature by application of magnetic fields, pressure, or through chemical doping. Unlike classical critical points, where the critical fluctuations are limited to a narrow region around the phase transition, the influence of a quantum critical point $(\mathrm{QCP})$ persists over a wide range of temperatures above the QCP, where exotic phenomena such as non-Fermi liquid behavior or unconventional superconductivity may emerge [1].

The one-dimensional (1D) transverse-field Ising model and its anisotropic generalization 1D transverse-field XXZ model are ideally suitable for the study of quantum criticalities, as this model is exactly solvable and the associated quantum states are highly field-tunable [1-7]. Recently, the quasi-1D spin-chain antiferromagnet $\mathrm{BaCo}_{2} \mathrm{~V}_{2} \mathrm{O}_{8}$, which can be well described by the 1D XXZ model with medium Ising-type anisotropy $(\Delta \simeq 2)$, has attracted great interest in the community [8-17]. $\mathrm{BaCo}_{2} \mathrm{~V}_{2} \mathrm{O}_{8}$ exhibits an easy-axis anisotropy along the $c$ axis (chain axis) and the weak interchain interaction leads to a 3D long-range Néel order below $T_{N} \simeq 5.5 \mathrm{~K}$. The 3D long-range magnetic order can be suppressed by a relatively weak transverse magnetic field of $10 \mathrm{~T}$ along the tetragonal $a / b$ direction $[9,10,12]$, as the transverse field can induce a perpendicular effective staggered field owing to the presence of the screw chain structure, where the the local easy axis of the $\mathrm{CoO}_{6}$ octahedra is tilted by $\simeq 4.8^{\circ}$ from the $c$ axis $[9,10,13,15]$.

\footnotetext{
*wangwb@fudan.edu.cn

${ }^{\dagger}$ zhaoj@fudan.edu.cn
}

It has been predicted theoretically that $E_{8}$ excitations, which are exotic bound states with emergent $E_{8}$ symmetry (a Lie algebra of rank 8), would appear in the vicinity of the QCP of the 1D transverse field Ising model perturbed by a longitudinal field $[4,18,19]$. The ratios of the masses of the eight $E_{8}$ particles are related to the roots of the $E_{8}$ algebra and can be determined analytically $[18,19]$. The first two meson particles of the $E_{8}$ excitations spectrum were indeed observed in the quasi-1D Ising ferromagnet $\mathrm{CoNb}_{2} \mathrm{O}_{6}[4,20]$. Very recently, inelastic neutron scattering (INS) and the terahertz spectroscopy measurements have revealed a series of sharp modes in $\mathrm{BaCo}_{2} \mathrm{~V}_{2} \mathrm{O}_{8}$ under applied field of $4.7 \mathrm{~T}$ along the tetragonal $b$ direction $[16,17]$. The energy ratios of these excitations are close to the expected value for $E_{8}$ excitations. These results were interpreted as evidence for a $1 \mathrm{D}$ quantum criticality at the field of $4.7 \mathrm{~T}$ in $\mathrm{BaCo}_{2} \mathrm{~V}_{2} \mathrm{O}_{8}[16,17]$.

The situation is less clear in the sister compound $\mathrm{SrCO}_{2} \mathrm{~V}_{2} \mathrm{O}_{8}$, as a recent nuclear magnetic resonance (NMR) measurement suggests the presence of a $1 \mathrm{D}$ QCP at $7.7 \mathrm{~T}$, which is above the 3D critical field of $7 \mathrm{~T}$ under applied fields along the $a$ direction [21]. At this field, the staggered longitudinal field induced by 3D magnetic order that is believed to be essential for the $E_{8}$ excitations is no longer present [17]. It is therefore particularly interesting to elucidate the evolution of the magnetic structure and spin excitations in transverse fields to reveal the nature of the underlying quantum phase transitions in $\mathrm{SrCo}_{2} \mathrm{~V}_{2} \mathrm{O}_{8}$.

\section{METHODS}

Our $\mathrm{SrCo}_{2} \mathrm{~V}_{2} \mathrm{O}_{8}$ single crystals were grown using the floating-zone method [22]. First, polycrystalline samples were 
synthesized by the standard solid state reaction method. Stoichiometric ratios of $\mathrm{SrCO}_{3}, \mathrm{CoC}_{2} \mathrm{O}_{4} \cdot 2 \mathrm{H}_{2} \mathrm{O}$, and $\mathrm{V}_{2} \mathrm{O}_{5}$ powders were mixed and ground in a mortar. The mixture was packed into an alumina crucible and calcined at $850{ }^{\circ} \mathrm{C}$ in air for $72 \mathrm{~h}$ with several intermediate grindings. The product was pressed into pellets and sintered at $900^{\circ} \mathrm{C}$ in air for $36 \mathrm{~h}$. The resulting powder was packed into latex tubes and pressed into rods with a diameter of $8 \mathrm{~mm}$ under $300 \mathrm{MPa}$ hydrostatic pressure. The obtained rods were sintered at $1000^{\circ} \mathrm{C}$ for $4 \mathrm{~h}$ in a vertical furnace. The resulting feed rods were then transferred into an optical floating zone furnace for the crystal growth. During the growth, the feed rod and the crystal were counter-rotated at 24.6 and $29.2 \mathrm{rpm}$, respectively, and the growth rate was set at $0.5 \mathrm{~mm} / \mathrm{h}$. High quality single crystals of $\mathrm{SrCo}_{2} \mathrm{~V}_{2} \mathrm{O}_{8}$ up to $10 \mathrm{~cm}$ in length can be grown in a flowing mixture of oxygen (20\%) and argon (80\%) at ambient pressure.

We have performed specific heat and DC magnetization measurements on small pieces of crystals cutting from large single crystals. The specific heat down to $0.4 \mathrm{~K}$ was measured in a Physical Property Measurement System (Quantum Design) with the adiabatic thermal relaxation technique. The temperature- and field-dependent magnetization was measured in a Physical Property Measurement System (Quantum Design) and a Superconducting Quantum Interference Device (Quantum Design) magnetometer, respectively.

The neutron scattering measurements were carried out on the SPINS cold triple-axis spectrometer, the DCS chopper time-of-flight spectrometer at the NIST Center for Neutron Research (NCNR), United States and the cold neutron multichopper spectrometer LET at the ISIS Facility, Rutherford Appleton Laboratory, Didcot, UK [23,24]. The same piece of $\mathrm{SrCo}_{2} \mathrm{~V}_{2} \mathrm{O}_{8}$ single crystal with the mass of $4.7 \mathrm{~g}$ was aligned in the $(H, 0, L)$ scattering plane and the magnetic fields were applied along the crystallographic $b$ direction (equivalent to the $a$ direction) for the SPINS and the DCS experiments. For the SPINS experiment, all data were measured at $1.5 \mathrm{~K}$ using the vertical focusing pyrolytic graphite monochromator and analyzer. A cold Be filter was installed after the sample and the final neutron energy was fixed at $5 \mathrm{meV}$. The collimation of Guide-20'-sample-20'-open was used and the energy resolution was $\sim 0.3 \mathrm{meV}$. For the DCS experiment, the data were measured at $1.5 \mathrm{~K}$ with the incident energies of 13.1 and $6.68 \mathrm{meV}$. The corresponding energy resolution is $\sim 0.63$ and $\sim 0.23 \mathrm{meV}$, respectively. For the LET experiment, two pieces of single crystals $(7.7 \mathrm{~g})$ were co-aligned in the $(H, K, 0)$ plane for the measurements. The measurements were carried out at $2 \mathrm{~K}$ with the incident neutron energies of 20.1, 7.0, 3.5, and $2.1 \mathrm{meV}$ [25]. The DCS and LET data were analyzed using the DAVE and Horace-MATLAB suite, respectively [26,27]. Wave vector $\mathbf{Q}$ is defined as $\mathbf{Q}=H \mathbf{a}^{*}+K \mathbf{b}^{*}+L \mathbf{c}^{*}$, where $H, K, L$ are Miller indices and $\mathbf{a}^{*}=\hat{\mathbf{a}} 2 \pi / a, \mathbf{b}^{*}=\hat{\mathbf{b}} 2 \pi / b$, $\mathbf{c}^{*}=\hat{\mathbf{c}} 2 \pi / c$ with $a=b=12.267 \AA, c=8.424 \AA$.

\section{RESULTS AND DISCUSSION}

$\mathrm{SrCo}_{2} \mathrm{~V}_{2} \mathrm{O}_{8}$ exhibits a tetragonal crystal structure with four screw chains of $\mathrm{Co}^{2+}$ ions propagating along the crystallographic $c$ direction per unit cell (Fig. 1, only two screw chains are plotted for simplicity). The magnetic chains are well sep-

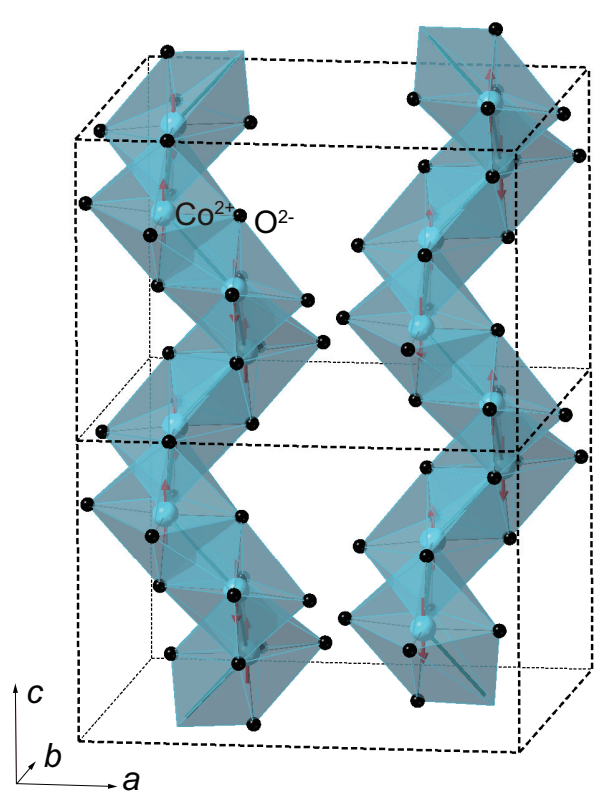

FIG. 1. Schematic of the lattice and magnetic structure for $\mathrm{SrCo}_{2} \mathrm{~V}_{2} \mathrm{O}_{8}$ at zero field. Only two chains in the unit cell are plotted for clarity, and the arrows indicate the magnetic moments below $T_{N}$.

arated by the nonmagnetic $\mathrm{V}^{5+}$ and $\mathrm{Sr}^{2+}$ ions. Combining the effect of spin-orbit coupling and the crystal electric fields in the $\mathrm{CoO}_{6}$ octahedra, the $\mathrm{Co}^{2+}$ ions exhibit the Kramers doublets ground state and can be well described by an effective spin- $1 / 2$ moment $[28,29]$. There is a subtle tilt $\left(\simeq 5.1^{\circ}\right)$ of the local easy axis of the $\mathrm{CoO}_{6}$ octahedra with respect to the $c$ direction in $\mathrm{SrCo}_{2} \mathrm{~V}_{2} \mathrm{O}_{8}$, and such tilt will lead to staggered fields along the $a$ and $c$ directions when applying a uniform field along the $b$ direction [10].

Figure 2 illustrates the magnetic susceptibility on our $\mathrm{SrCo}_{2} \mathrm{~V}_{2} \mathrm{O}_{8}$ single crystal under magnetic field $H=1 \mathrm{~T}$ applied along the $b$ and $c$ directions between 2 and $300 \mathrm{~K}$. The susceptibility with $H / / c$ exhibits a broad hump feature at $\sim 40 \mathrm{~K}$ followed by an abrupt drop at $\sim 5 \mathrm{~K}$. When the

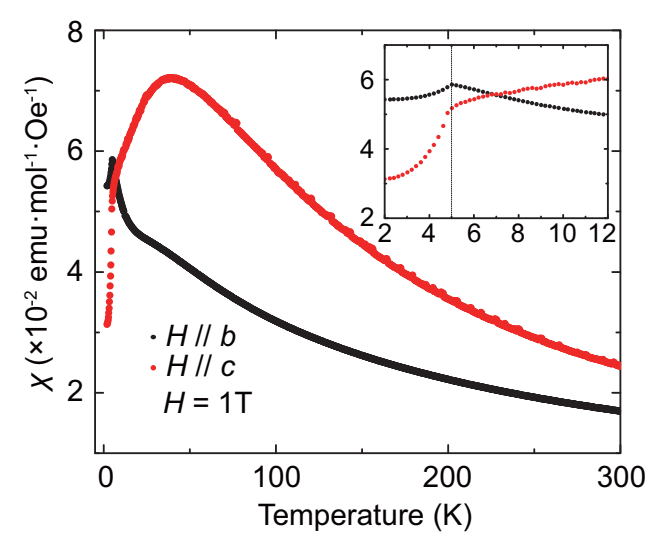

FIG. 2. Magnetic susceptibility measurements on $\mathrm{SrCo}_{2} \mathrm{~V}_{2} \mathrm{O}_{8}$ single crystal under magnetic field $H=1 \mathrm{~T}$ applied along the $b$ and $c$ directions. The inset shows the magnetic susceptibility curves near the transition temperature. The vertical line at $5 \mathrm{~K}$ indicates the transition temperature. 


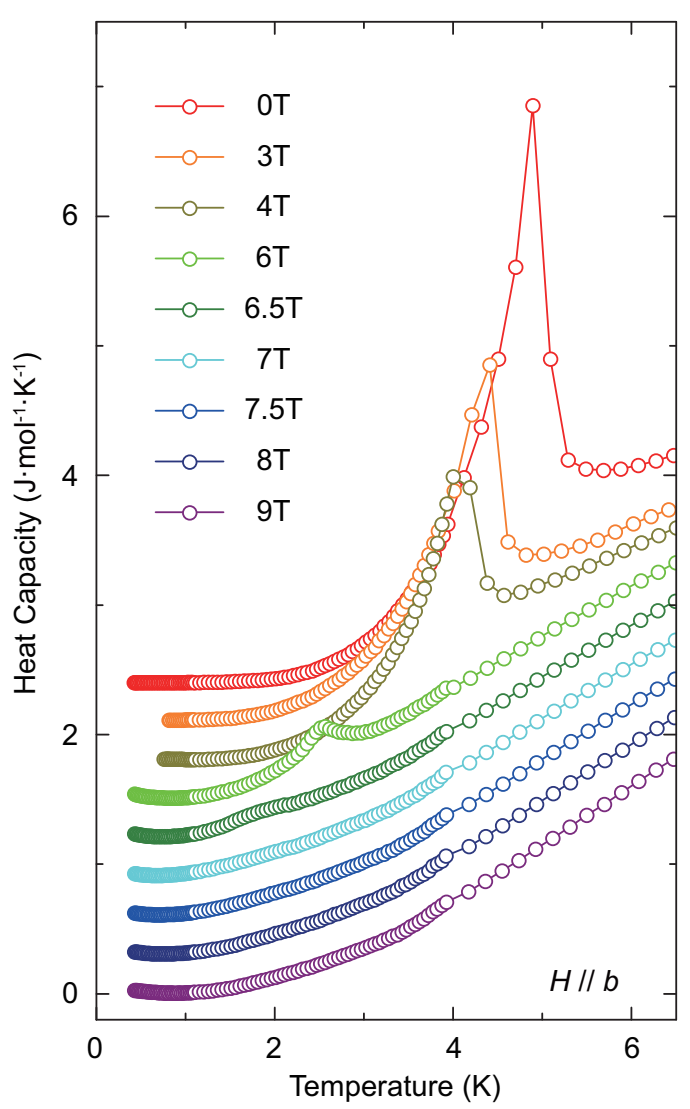

FIG. 3. Heat capacity measurements on $\mathrm{SrCo}_{2} \mathrm{~V}_{2} \mathrm{O}_{8}$ single crystal under magnetic fields along the $b$ direction. An offset has been added in each heat capacity curve for clarity, and the heat capacity data are raw data without background subtraction.

magnetic field is applied along the $b$ direction, a sharp peak in susceptibility is observed at $\sim 5 \mathrm{~K}$. The heat capacity curve also shows sharp anomalies at $\sim 5 \mathrm{~K}$ in zero field, indicative of a magnetic phase transition [Fig. 3]. These results are broadly consistent with previous reports [30,31]. The anomalies in heat capacity are progressively suppressed by transverse fields applied along the $b$ direction and eventually vanish at the critical field of $7 \mathrm{~T}$ [Fig. 3].

Figure 4 shows the magnetization as a function of a transverse field, which exhibits weak anomalies near the phase transition. The anomalies can be seen more clearly in the field derivative of the magnetization curves [Fig. 4]. This, together with the heat capacity measurements, results in the phase diagram shown in Fig. 5. On the other hand, we have not observed any anomalies in magnetization or heat capacity near 7.7 $\mathrm{T}$ where previous NMR measurements suggested the presence of a 1D QCP [21].

In order to elucidate the evolution of the magnetic phase transitions seen in heat capacity and magnetization measurements, we used neutron scattering to measure the structural and magnetic ordering properties in $\mathrm{SrCo}_{2} \mathrm{~V}_{2} \mathrm{O}_{8}$ in transverse fields. It is shown that the magnetic Bragg peak $\mathbf{Q}=(2$, $0,1)$ associated with the 3D Néel-type magnetic long-range order in zero field is gradually suppressed by the increasing transverse field, leading to a critical point at $7 \mathrm{~T}$ [Figs. 6(a) and 6(b)]. On the other hand, the intensities of the Bragg peak at $\mathbf{Q}=(1,0,1)$ and equivalent positions actually increase with

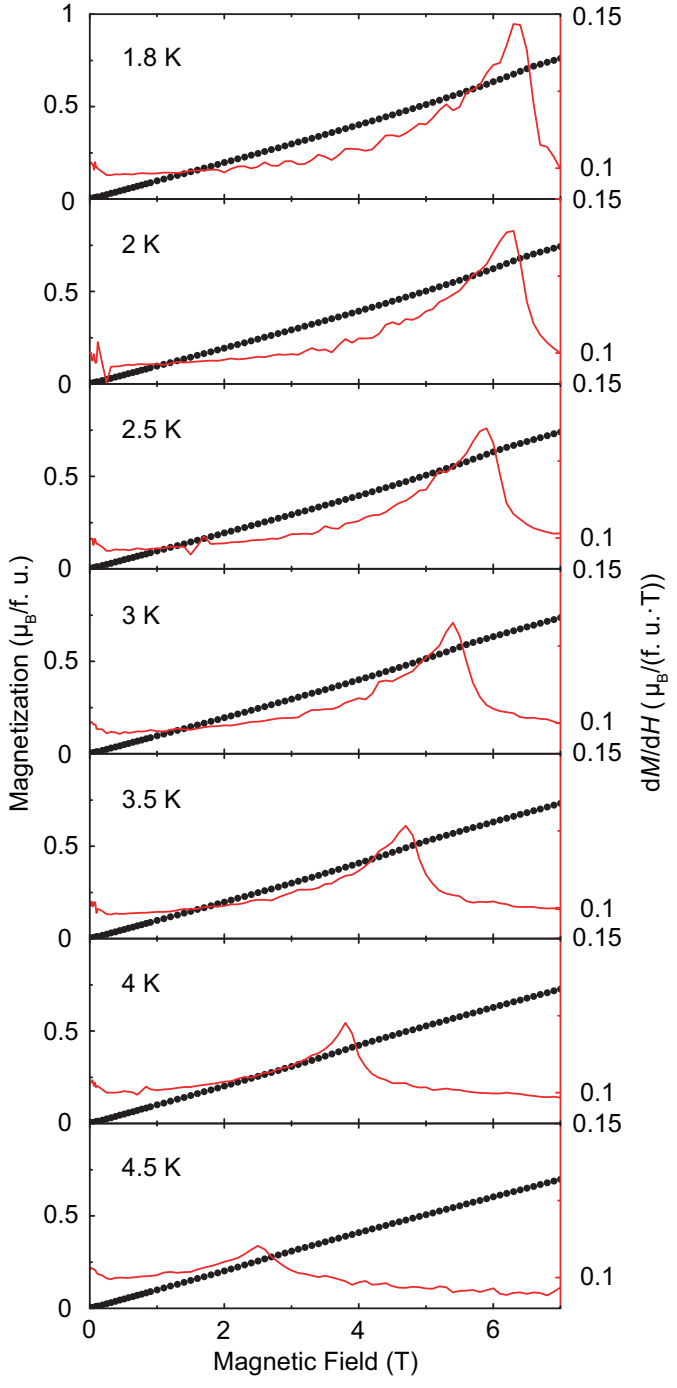

FIG. 4. Field dependence of the magnetization of $\mathrm{SrCo}_{2} \mathrm{~V}_{2} \mathrm{O}_{8}$ single crystal measured at various temperatures. The field is applied along the $b$ direction. The red lines denote the field derivations of the magnetization curves.

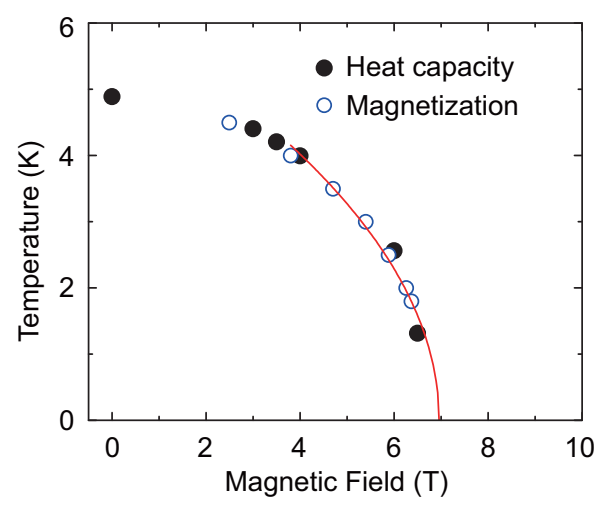

FIG. 5. Phase diagram of $\mathrm{SrCo}_{2} \mathrm{~V}_{2} \mathrm{O}_{8}$ under transverse fields along the $b$ direction. The solid black circles represent the Néel temperature $T_{N}$ determined by the heat capacity measurements, and the hollow blue circles represent the critical fields determined by the magnetization curves. The red line is a function fit to $T \sim$ $\left(H-H_{c}\right)^{0.5}$ with the critical field of $H_{c} \approx 6.96 \pm 0.08 \mathrm{~T}$. 

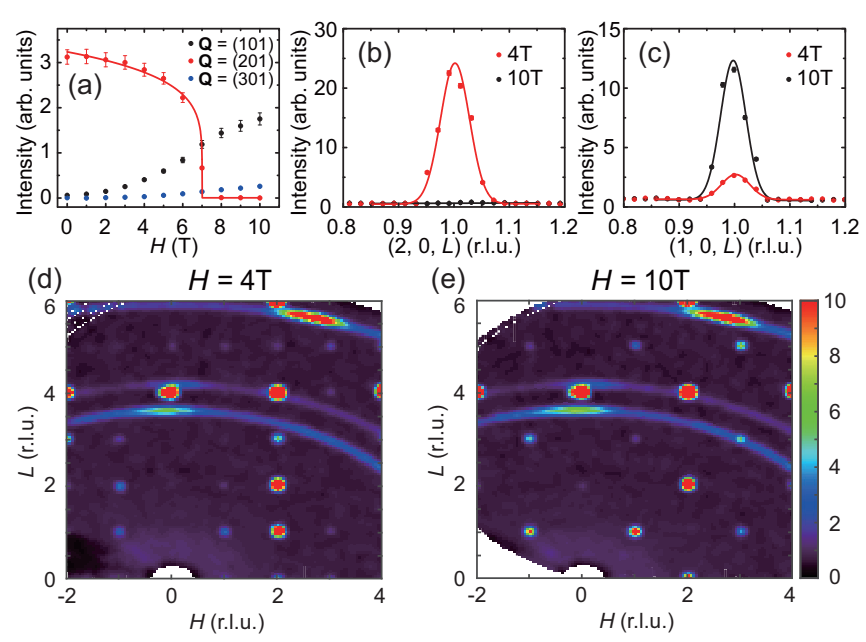

(e)

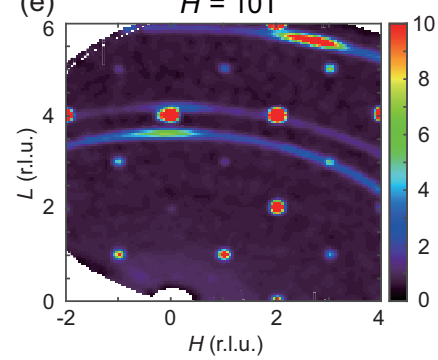

FIG. 6. (a) Field dependence of the neutron diffraction intensity at the indicated $\mathbf{Q}$ positions at $1.5 \mathrm{~K}$. The red line is a fit to $I \sim$ $\left(H-H_{c}\right)^{2 \beta}$ with the critical field of $H_{c} \approx 7.001 \pm 0.006 \mathrm{~T}$ and the critical exponent of $2 \beta \approx 0.18 \pm 0.02$. The data were collected at SPINS. (b) Q-cuts across $(2,0,1)$ along the $L$ direction at 4 and $10 \mathrm{~T}$ at $E=0 \mathrm{meV}$. (c) $\mathbf{Q}$-cuts across $(1,0,1)$ along the $L$ direction at 4 and $10 \mathrm{~T}$ at $E=0 \mathrm{meV}$. (d), (e) Elastic constant-energy slice obtained at $1.5 \mathrm{~K}$ at 4 and $10 \mathrm{~T}$, respectively. The color bar indicates scattering intensity in arbitrary units in linear scale, r.l.u., reciprocal lattice units. From (b) to (e), the data were collected at DCS.

increasing field, indicative of a new field-induced magnetic structure [Figs. 6(a) and 6(c)].

To determine the evolution of the magnetic structures with field, we mapped out the neutron diffraction intensities in various magnetic fields [Figs. 6(d) and 6(e)]. It is shown that the nuclear Bragg peaks measured at zero field can be fitted to a crystal structure with the space group $\mathrm{I} 4_{1} \mathrm{~cd}$ (No. 110). The magnetic peaks measured at zero field are indexed to the wave vector $\mathbf{k}=(0,0,1)$ and fitted to magnetic structure consisting of $1 \mathrm{D}$ antiferromagnetic chains along the $c$ axis. Inter-chain correlations are ferromagnetic along the $a$ - (or $b$-) direction and antiferromagnetic along the $b$ - (or $a$-) direction, resulting in two magnetic twin domains that can be described by magnetic space group $\mathrm{P}_{I} \mathrm{ca} 2{ }_{1}$ (BNS No. 29.110). The refinement yields an ordered magnetic moment of $m_{c}=2.23(6) \mu_{B}$ where the ratio of the two magnetic domains are 46(2):54(2). The magnetic structure in zero field is broadly consistent with the Néel-type magnetic order reported in previous work [22,32].

Figure 6(e) illustrates the diffraction pattern at $10 \mathrm{~T}$ above the critical field of $7 \mathrm{~T}$. The refined magnetic structure with propagation vector $\mathbf{k}=(0,0,0)$ is shown in Fig. $7(\mathrm{~b})$. The moment along the $c$ axis is largely suppressed, while the magnetic moments along the $a$ and $b$ directions are enhanced. The magnetic structure consists of antiferromagnetic moments along the $a$ axis induced by the staggered field and ferromagnetic moments along the $b$ axis induced by the uniform transverse field. The antiferromagnetic moments adopt the magnetic space group $\mathrm{I} 4_{1} \mathrm{c}^{\prime} \mathrm{d}^{\prime}$ (BNS No. 110.249), with $m_{a}=0.86(6) \mu_{B}, m_{b}=0.58(5) \mu_{B}$.

The magnetic structure is even more complicated at $4 \mathrm{~T}$, below the critical field. The diffraction data reveal that both
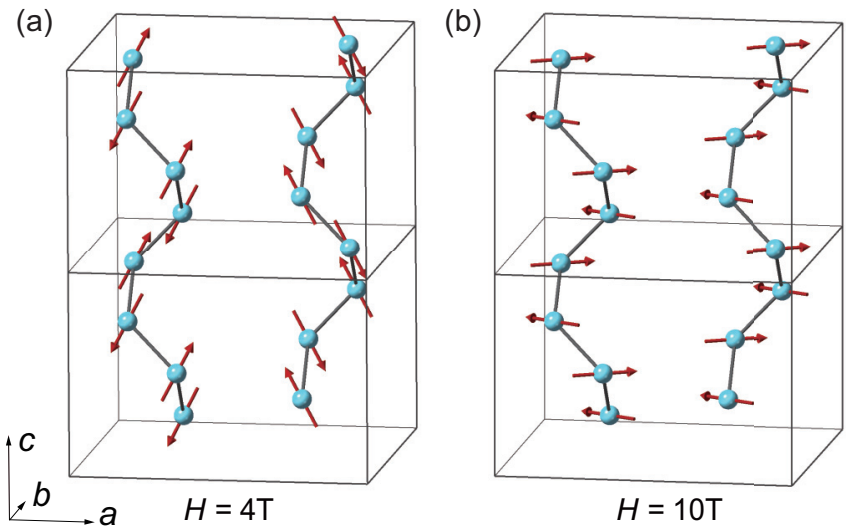

FIG. 7. (a) Schematic of the magnetic structure of $\mathrm{SrCo}_{2} \mathrm{~V}_{2} \mathrm{O}_{8}$ under transverse fields of $4 \mathrm{~T}$. Only two chains in the unit cell are plotted for clarity, and the blue balls represent the $\mathrm{Co}^{2+}$ ions. (b) Schematic of the magnetic structure of $\mathrm{SrCo}_{2} \mathrm{~V}_{2} \mathrm{O}_{8}$ under transverse fields of $10 \mathrm{~T}$.

wave vectors $\mathbf{k}=(0,0,0)$ and $\mathbf{k}=(0,0,1)$ show magnetic intensities at $4 \mathrm{~T}$. The refinements show that the magnetic moments are antiferromagnetically aligned within the chain. The moments along the $c$ axis are antiferromagnetically aligned between equivalent chains, whereas the moments along the $a$ axis are ferromagnetically aligned between equivalent chains [Fig. 7(a)]. In addition, all moments are slightly canted toward the $b$ axis due to the transverse field. The refined magnetic moments are $m_{a}=0.40(6) \mu_{B}, m_{b}=0.27(5) \mu_{B}$, and $m_{c}=2.0(2) \mu_{B}$. The complicated noncollinear magnetic structure is apparently owing to the competition between the intrinsic magnetic interactions and the external transverse field/staggered field in the intermediate field regime. Figure 8 summarizes the nuclear and magnetic structure refinements in
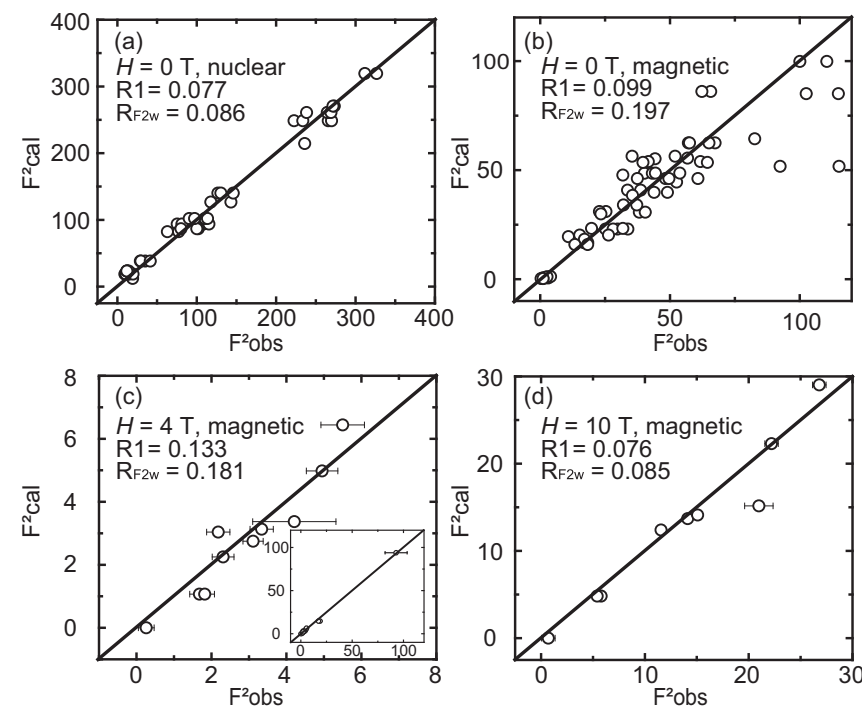

FIG. 8. Comparison of the squared magnetic structure factors between observations and calculations. (a) Nuclear peaks measured at zero field. (b) Magnetic peaks measured at zero field. (c) Magnetic peaks measured at $H=4 \mathrm{~T}$. The inset shows stronger magnetic peaks at the wave vector $\mathbf{k}=(0,0,1)$. (d) Magnetic peaks measured at $H=10 \mathrm{~T}$. The agreement factor is indicated on each panel. 

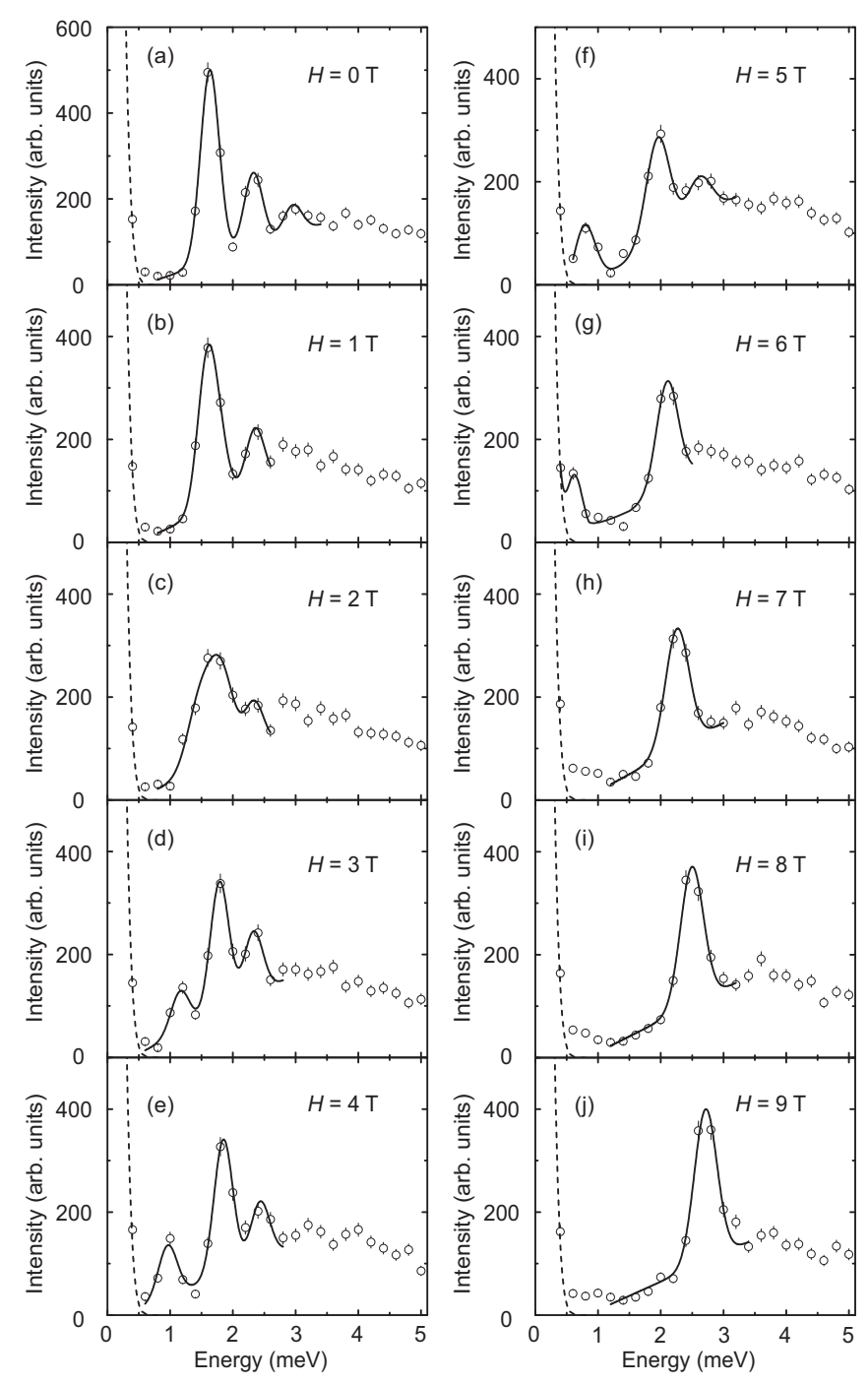

FIG. 9. Energy scans of the spin excitations in $\mathrm{SrCo}_{2} \mathrm{~V}_{2} \mathrm{O}_{8}$ at indicated transverse fields at $\mathbf{Q}=(0,0,2)$ and $1.5 \mathrm{~K}$. The solid lines are the Gaussian fits of the data. The dashed lines indicate the elastic incoherent scattering. The data were collected at SPINS.

various transverse fields along the $b$ direction, showing good agreement between observations and calculations.

Having established evolution of the magnetic structure with field, we now turn to the quantum excitations associated with the observed magnetic structure. In contrast with conventional spin wave like excitations, the spin excitation spectra of $\mathrm{SrCO}_{2} \mathrm{~V}_{2} \mathrm{O}_{8}$ show a series of discrete modes at zero field below $T_{N}$ [Fig. 9(a)], which can be interpreted as spinons confined by the inter-chain couplings induced linear potential [11,33-35]. At least 3 peaks at energies of $1.63 \mathrm{meV}$, $2.32 \mathrm{meV}, 2.95 \mathrm{meV}$ can be resolved at $(0,0,2)$, which are in agreement with previous INS and terahertz spectroscopy measurements $[29,36]$. Interestingly, the discrete sharp modes persist in applied magnetic fields, although their intensities and energies are strongly modified [Figs. 9(b)-9(j)]. The lowest energy mode gradually broadened with increasing transverse magnetic field up to $2 \mathrm{~T}$ and then split into two modes at higher fields. The low energy mode reaches a minimum energy at $\sim 7 \mathrm{~T}$, which corresponds to the $3 \mathrm{D} \mathrm{QCP}$ associated

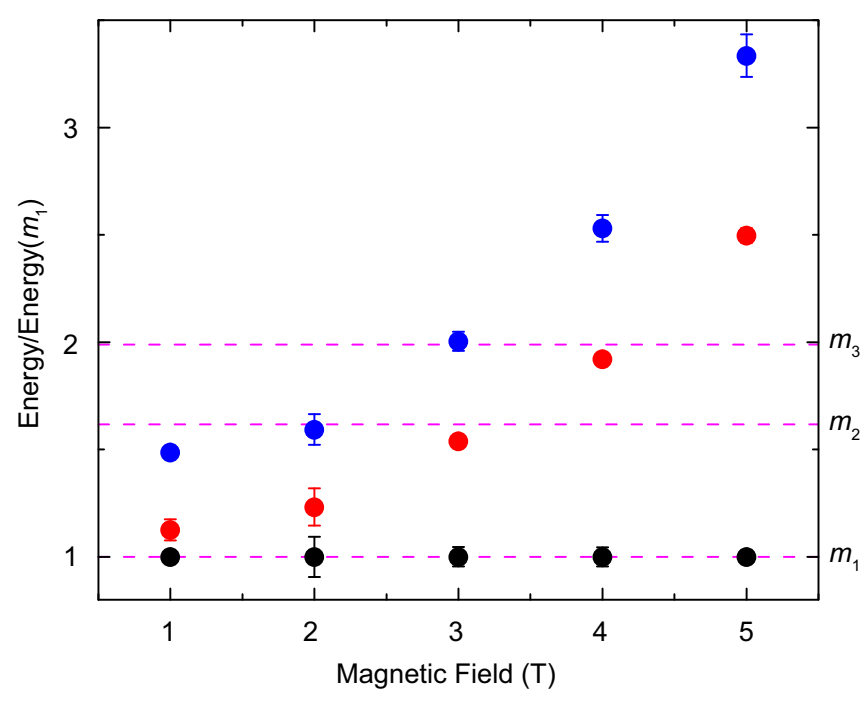

FIG. 10. The energy ratios of high energy excitation modes with respect to the lowest energy mode in $\mathrm{SrCo}_{2} \mathrm{~V}_{2} \mathrm{O}_{8}$. The black, red, and blue dots indicate the energy ratios of the first, second, and third peaks fitted from the energy scan measured at each field, respectively. The dashed lines indicate the theoretically expected ratios for the $E_{8}$ excitations adopted from Refs. [16,18].

with the suppression of the Néel-type magnetic ordering moment [37]. This is consistent with our specific heat and magnetization measurements [Figs. 3 and 4]. On the other hand, the upper branches of the spin excitations move to higher energies as the magnetic field increases. In magnetic fields above the critical field of $7 \mathrm{~T}$, only one sharp mode is discernible.

Figure 10 summarizes the energy ratios of high energy excitation modes with respect to the lowest energy mode in $\mathrm{SrCo}_{2} \mathrm{~V}_{2} \mathrm{O}_{8}$. It is shown that the energy ratios increase monotonically with increasing field. Interestingly, at $3 \mathrm{~T}$, the energy ratios are close to the expected values of the $E_{8}$ excitations, similar to those observed in $\mathrm{BaCo}_{2} \mathrm{~V}_{2} \mathrm{O}_{8}$ near $4.7 \mathrm{~T}[16,17]$. In addition, the intensity ratios of the observed modes in $\mathrm{SrCo}_{2} \mathrm{~V}_{2} \mathrm{O}_{8}$ are also close to those of $\mathrm{BaCo}_{2} \mathrm{~V}_{2} \mathrm{O}_{8}$. In both samples, the peak intensity of $m_{1}$ is lower than that of $m_{2}$, which was attributed to the spin exchange anisotropy [17]. If the observed discrete modes are indeed due to $E_{8}$ excitations, it suggests the presence of a $1 \mathrm{D} \mathrm{QCP}$ at $\sim 3 \mathrm{~T}$ in $\mathrm{SrCo}_{2} \mathrm{~V}_{2} \mathrm{O}_{8}$. We note that the critical field of $\mathrm{SrCo}_{2} \mathrm{~V}_{2} \mathrm{O}_{8}$ is slightly lower than that $(4.7 \mathrm{~T})$ of $\mathrm{BaCo}_{2} \mathrm{~V}_{2} \mathrm{O}_{8}$, which is not surprising, as the $3 \mathrm{D}$ quantum critical field of the former is also lower. This could be simply due to the fact that the tilting angle of the local easy axis of the $\mathrm{CoO}_{6}$ octahedra and the resulting effective staggered field in $\mathrm{SrCo}_{2} \mathrm{~V}_{2} \mathrm{O}_{8}$ are larger than those of $\mathrm{BaCo}_{2} \mathrm{~V}_{2} \mathrm{O}_{8}$. However, this critical field is significantly lower than that $(7.7 \mathrm{~T})$ implied by a recent NMR measurement [21]. These results suggest that the quantum critical behavior in $\mathrm{SrCo}_{2} \mathrm{~V}_{2} \mathrm{O}_{8}$ is more complicated than expected. Further studies are required to elucidate the relationship between the possible multiple QCPs in this compound.

\section{CONCLUSIONS}

To conclude, we have studied the evolution of the magnetic ordering properties and spin excitations in the quasi-1D quan- 
tum magnet $\mathrm{SrCo}_{2} \mathrm{~V}_{2} \mathrm{O}_{8}$ in transverse fields up to $10 \mathrm{~T}$. We show that the magnetic structure exhibits unconventional spin reorientation transitions due to the applied transverse field and staggered field. The INS and thermodynamic measurements consistently show a 3D QCP at $7 \mathrm{~T}$, which is associated with the suppression of the Néel-type magnetic ordering moment revealed by neutron diffraction measurements. A series of discrete sharp excitation modes are observed in zero field, which evolve with field in a similar manner as those of $\mathrm{BaCo}_{2} \mathrm{~V}_{2} \mathrm{O}_{8}$. At $3 \mathrm{~T}$, the energy ratios are close to the expected values of the $E_{8}$ excitations. If these discrete modes are associated with the $E_{8}$ excitations, it would suggest that the $1 \mathrm{D}$ quantum critical field is much lower than that $(7.7 \mathrm{~T})$ implied by a recent NMR measurement, indicating a rather complex QCP behavior in this compound.

The data sets for the neutron scattering experiment on the time-of-flight LET spectrometer are available from the ISIS facility, Rutherford Appleton Laboratory data portal [25]. All other data that support the plots within this paper and other findings of this study are available from the corresponding authors on reasonable request.

\section{ACKNOWLEDGMENTS}

This work was partially supported by the National Key R\&D Program of the MOST of China (Grant No. 2016YFA0300203), the Innovation Program of Shanghai Municipal Education Commission (Grant No. 2017-01-07-0007-E00018), the Shanghai Municipal Science and Technology Major Project (Grant No. 2019SHZDZX01), and the National Natural Science Foundation of China (Grant No. 11874119). The identification of any commercial product or trade name does not imply endorsement or recommendation by the National Institute of Standards and Technology.
[1] S. Sachdev, Quantum Phase Transitions, 2nd ed. (Cambridge University Press, Cambridge, 2011).

[2] D. Bitko, T. F. Rosenbaum, and G. Aeppli, Phys. Rev. Lett. 77, 940 (1996).

[3] M. Kenzelmann, R. Coldea, D. A. Tennant, D. Visser, M. Hofmann, P. Smeibidl, and Z. Tylczynski, Phys. Rev. B 65, 144432 (2002).

[4] R. Coldea, D. A. Tennant, E. M. Wheeler, E. Wawrzynska, D. Prabhakaran, M. Telling, K. Habicht, P. Smeibidl, and K. Kiefer, Science 327, 177 (2010).

[5] O. Breunig, M. Garst, E. Sela, B. Buldmann, P. Becker, L. Bohatý, R. Müller, and T. Lorenz, Phys. Rev. Lett. 111, 187202 (2013).

[6] O. Breunig, M. Garst, A. Klümper, J. Rohrkamp, M. M. Turnbull, and T. Lorenz, Sci. Adv. 3, eaao3773 (2017).

[7] D. Blosser, V. K. Bhartiya, D. J. Voneshen, and A. Zheludev, Phys. Rev. Lett. 121, 247201 (2018).

[8] S. Kimura, H. Yashiro, K. Okunishi, M. Hagiwara, Z. He, K. Kindo, T. Taniyama, and M. Itoh, Phys. Rev. Lett. 99, 087602 (2007).

[9] S. Kimura, K. Okunishi, M. Hagiwara, K. Kindo, Z. He, T. Taniyama, M. Itoh, K. Koyama, and K. Watanabe, J. Phys. Soc. Jpn. 82, 033706 (2013).

[10] S. K. Niesen, O. Breunig, S. Salm, M. Seher, M. Valldor, P. Warzanowski, and T. Lorenz, Phys. Rev. B 90, 104419 (2014).

[11] B. Grenier, S. Petit, V. Simonet, E. Canévet, L.-P. Regnault, S. Raymond, B. Canals, C. Berthier, and P. Lejay, Phys. Rev. Lett. 114, 017201 (2015).

[12] A. Okutani, S. Kimura, T. Takeuchi, and M. Hagiwara, Appl. Magn. Reson. 46, 1003 (2015).

[13] M. Matsuda, H. Onishi, A. Okutani, J. Ma, H. Agrawal, T. Hong, D. M. Pajerowski, J. R. D. Copley, K. Okunishi, M. Mori et al., Phys. Rev. B 96, 024439 (2017).

[14] Z. Wang, T. Lorenz, D. I. Gorbunov, P. T. Cong, Y. Kohama, S. Niesen, O. Breunig, J. Engelmayer, A. Herman, J. Wu et al., Phys. Rev. Lett. 120, 207205 (2018).
[15] Q. Faure, S. Takayoshi, S. Petit, V. Simonet, S. Raymond, L.-P. Regnault, M. Boehm, J. S. White, M. Månsson, C. Rüegg et al., Nat. Phys. 14, 716 (2018).

[16] Z. Zhang, K. Amelin, X. Wang, H. Zou, J. Yang, U. Nagel, T. Rõõm, T. Dey, A. A. Nugroho, T. Lorenz, J. Wu, and Z. Wang, Phys. Rev. B 101, 220411(R) (2020).

[17] H. Zou, Y. Cui, X. Wang, Z. Zhang, J. Yang, G. Xu, A. Okutani, M. Hagiwara, M. Matsuda, G. Wang et al., arXiv:2005.13302.

[18] A. B. Zamolodchikov, Int. J. Mod. Phys. A 4, 4235 (1989).

[19] J. Wu, M. Kormos, and Q. Si, Phys. Rev. Lett. 113, 247201 (2014).

[20] K. Amelin, J. Engelmayer, J. Viirok, U. Nagel, T. Rõõm, T. Lorenz, and Z. Wang, Phys. Rev. B 102, 104431 (2020).

[21] Y. Cui, H. Zou, N. Xi, Z. He, Y. X. Yang, L. Shu, G. H. Zhang, Z. Hu, T. Chen, R. Yu et al., Phys. Rev. Lett. 123, 067203 (2019).

[22] A. K. Bera, B. Lake, W.-D. Stein, and S. Zander, Phys. Rev. B 89, 094402 (2014).

[23] J. R. D. Copley and J. C. Cook, Chem. Phys. 292, 477 (2003).

[24] R. I. Bewley, J. W. Taylor, and S. M. Bennington, Nucl. Instrum. Methods Phys. Res., Sect. A 637, 128 (2011).

[25] J. Zhao et al., Exotic spin excitations in a quasi one-dimensional anisotropic Heisenberg magnet, STFC ISIS Neutron and Muon Source, doi: 10.5286/ISIS.E.RB1810289 (2018).

[26] R. T. Azuah, L. R. Kneller, Y. Qiu, P. L. W. Tregenna-Piggott, C. M. Brown, J. R. D. Copley, and R. M. Dimeo, J. Res. Natl. Inst. Stand. Technol. 114, 341 (2009).

[27] R. A. Ewings, A. Buts, M. D. Le, J. van Duijn, I. Bustinduy, and T. G. Perring, Nucl. Instrum. Methods Phys. Res., Sect. A 834, 132 (2016).

[28] M. E. Lines, Phys. Rev. 131, 546 (1963).

[29] Z. Wang, M. Schmidt, A. K. Bera, A. T. M. N. Islam, B. Lake, A. Loidl, and J. Deisenhofer, Phys. Rev. B 91, 140404(R) (2015). 
[30] Z. He, T. Taniyama, and M. Itoh, J. Cryst. Growth 293, 458 (2006).

[31] Z. He, T. Taniyama, and M. Itoh, Phys. Rev. B 73, 212406 (2006).

[32] J. Liu, J. Wang, W. Luo, J. Sheng, Z. He, S. A. Danilkin, and W. Bao, Chin. Phys. Lett. 33, 037502 (2016).

[33] H. Shiba, Prog. Theor. Phys. 64, 466 (1980).

[34] B. Lake, A. M. Tsvelik, S. Notbohm, D. A. Tennant, T. G. Perring, M. Reehuis, C. Sekar, G. Krabbes, and B. Büchner, Nat. Phys. 6, 50 (2010).

[35] C. M. Morris, R. Valdes Aguilar, A. Ghosh, S. M. Koohpayeh, J. Krizan, R. J. Cava, O. Tchernyshyov,
T. M. McQueen, and N. P. Armitage, Phys. Rev. Lett. 112, 137403 (2014).

[36] A. K. Bera, B. Lake, F. H. L. Essler, L. Vanderstraeten, C. Hubig, U. Schollwöck, A. T. M. N. Islam, A. Schneidewind, and D. L. Quintero-Castro, Phys. Rev. B 96, 054423 (2017).

[37] Z. Wang, J. Wu, S. Xu, W. Yang, C. Wu, A. K. Bera, A. T. M. Nazmul Islam, B. Lake, D. Kamenskyi, P. Gogoi, H. Engelkamp, N. Wang, J. Deisenhofer, and A. Loidl, Phys. Rev. B 94, 125130 (2016).

Correction: The middle initial of the ninth author was missing and has been fixed. 